\title{
Set-Membership Filtering Subject to Impulsive Measurement Outliers: A Recursive Algorithm
}

\author{
Lei Zou, Zidong Wang, Fellow, IEEE, Hang Geng, and Xiaohui Liu
}

\begin{abstract}
This paper is concerned with the set-membership filtering problem for a class of linear time-varying systems with norm-bounded noises and impulsive measurement outliers. A new representation is proposed to model the measurement outlier by an impulsive signal whose minimum interval length (i.e. the minimum duration between two adjacent impulsive signals) and minimum norm (i.e. the minimum of the norms of all impulsive signals) are larger than certain thresholds that are adjustable according to engineering practice. In order to guarantee satisfactory filtering performance, a so-called parameter-dependent set-membership filter is put forward that is capable of generating a time-varying ellipsoidal region containing the true system state. First, a novel outlier detection strategy is developed, based on a dedicatedly constructed input-output model, to examine whether the received measurement is corrupted by an outlier. Then, through the outcome of the outlier detection, the gain matrix of the desired filter and the corresponding ellipsoidal region are calculated by solving two recursive difference equations. Furthermore, the ultimate boundedness issue on the time-varying ellipsoidal region is thoroughly investigated. Finally, a simulation example is provided to demonstrate the effectiveness of our proposed parameter-dependent set-membership filtering strategy.
\end{abstract}

Index Terms-Set-membership filtering; Impulsive measurement outliers; Time-varying systems; Parameter-dependent filter; Boundedness analysis.

\section{INTRODUCTION}

The past few decades have witnessed a surge of research interest in a filtering problem that serves as a core topic in control and signal processing communities [1]-[14]. So far, a variety of filtering techniques have been developed and further applied in many practical areas such as system guidance and navigation, target tracking, process control, and fault detection. In general, the extensively studied filtering strategies can be divided into five categories, namely, minimum meansquared error filtering (e.g. the well-known Kalman filtering and extended Kalman filtering) [15]-[17], $\mathcal{H}_{\infty}$ filtering [18], [19], ultimately bounded filtering [20], [21], moving-horizon estimation [22]-[24] and set-membership filtering (or setvalued filtering) algorithms [25], [26]. For instance, in [27], a distributed resilient filter has been proposed for power systems

This work was supported in part by the National Natural Science Foundation of China under Grants 61703245, 61873148 and 61933007, the China Postdoctoral Science Foundation under Grant 2018T110702, the Postdoctoral Special Innovation Foundation of of Shandong province of China Grant 201701015, the European Union's Horizon 2020 Research and Innovation Programme under Grant 820776 (INTEGRADDE), the Royal Society of the UK, and the Alexander von Humboldt Foundation of Germany. (Corresponding author: Zidong Wang)

L. Zou, Z. Wang, H. Geng and X. Liu are with the Department of Computer Science, Brunel University London, Uxbridge, Middlesex, UB8 3PH, United Kingdom. (Email: Zidong.Wangebrunel.ac.uk). under denial-of-service attacks. The recursive filtering problem has been studied in [28] for time-delayed nonlinear stochastic systems with missing measurements, uniform quantization and Round-Robin protocol scheduling. In [29], a distributed $\mathcal{H}_{\infty}$-consensus filter has been designed for discrete timevarying systems subject to multiplicative noises and censored measurements over sensor networks. Among others, the setmembership filtering (SMF) scheme is particularly suitable for time-varying systems with unknown-but-bounded noises.

The original idea of SMF dates back to 1968 in [30] where a confidence region containing the true system state (rather than a concrete state estimate) is generated at each time instant. Compared with the pointwise filters (e.g. the minimum mean-squared-error filter, $\mathcal{H}_{\infty}$ filter and moving-horizon estimator), the set-membership filter utilizes the interval-based techniques. So far, the SMF problem has gained an ongoing research interest for various systems, e.g. [31]-[35] and the references therein. For example, in [33], a so-called probabilityguaranteed set-membership filter has been designed for timevarying systems with incomplete measurements, where the estimation error is contained in an ellipsoidal set with a given probability. The distributed SMF problem has been studied in [31] for linear time-varying systems with dynamic event-triggered transmission scheme. Among various SMF approaches, the recursive linear matrix inequality (RLMI) algorithm has proven to be popular, with which the filter parameter is recursively computed to confine the filtering error into a time-varying ellipsoidal region in the state-space. The RLMI-based approach is particularly suitable for online applications. Nevertheless, the ultimate boundedness of the time-varying ellipsoidal region remains to be an open yet crucial issue in quantifying the filtering performance. As such, it is of practical importance to develop new SMF techniques with a special focus on analyzing the ultimate boundedness.

Up to now, almost all SMF-related results have been exclusively concerned with bounded disturbances, where the size of the corresponding ellipsoidal region (containing the true system state) is largely dependent on the bounds of the underlying noises, which implies that the filtering performance would deteriorate if the amplitudes of external disturbances are out of a normal range. Note that, in many practical applications, the system measurements might suffer from largeamplitude disturbances, leading to the so-called measurement outliers. Compared with other extensively investigated noises, the measurement outliers have their own characteristics of i) occasional/intermittent/probabilistic occurrences and ii) unexpectedly large amplitudes. Till now, some initial results have been reported in the literature on the filtering problem with 
outliers, e.g. [36]-[42] and the references therein.

There are generally two frameworks for dealing with the filtering problem subject to outliers, namely, the passive robustness-based framework and the active detection-based framework. In the passive resistance-based framework, the filter is designed by selecting suitable filter parameters based on statistical tests or on-line adaptive adjustment such that the filtering performance is less sensitive to abnormal noises (outliers) in a statistical/saturated sense [38], [40], [43]. For example, in [44], [45], some robust filters have been developed to reduce the sensitivity of the filtering performance to outliers. In [38], a so-called stubborn state observer has been constructed by using the saturated innovation in the observer design, under which the effects induced by the possible outliers would be restrained. Nevertheless, the passive robustnessbased schemes cannot guarantee the complete elimination of the effects induced by the measurement outliers in the filtering process. In the active detection-based framework, the filter is designed with dedicatedly proposed structure with hope to cancel the innovations corrupted by outliers. For example, in [37], a novel moving-horizon estimator has been developed to cope with the estimation problem subject to outliers, where a special leave-one-out method has been employed to identify the "harmful measurements" possibly contaminated by outliers. Unfortunately, to the best of the authors' knowledge, the SMF problem subject to measurement outliers has not gained adequate research attention yet, despite the critical importance of mitigating the outlier-induced effects. It is, therefore, the main motivation of this paper to fill such a gap.

Summarizing the discussions made thus far, there is a practical need to deal with the SMF problem for time-varying systems with measurement outliers. Some essential difficulties we have to face are identified as follows: 1) how to establish reasonable model for the measurement outlier according to engineering practice; 2) how to distinguish the measurement outputs contaminated by outliers from those normal measurements? 3) how to design the set-membership filter that prevents the filtering performance from being degraded by the measurement outliers? and 4) how to deal with the ultimate boundedness analysis issue on the time-varying ellipsoidal region containing the true system state?

In response to the identified difficulties, the contributions of this paper are highlighted as follows: 1) the measurement outlier is modeled by an impulsive signal with its interval length (i.e. the minimum duration between two adjacent impulsive signals) and minimum norm (i.e. the minimum of the norms of all impulsive signals) determined by engineering practice; 2) the SMF problem is, for the first time, investigated for time-varying systems with measurement outliers where a novel detection method is developed to examine whether the current measurement output is corrupted by an outlier; 3) a dedicatedly designed parameter-dependent set-membership (PDSM) filter is employed to "discard" the measurements corrupted by outliers; and 4) the ultimate boundedness is investigated for the time-varying ellipsoidal region containing the true system state.

The remainder of this paper is organized as follows. In Section II, the time-varying system with impulsive measure- ment outliers is introduced and the corresponding PDSM filter structure is proposed. In Section III, the detection strategy of the measurement outlier is developed, and the desired timevarying filter gain matrix is calculated by solving two recursive difference equations. Then, the boundedness analysis of the constraint matrices concerning the ellipsoidal region is carried out. A numerical simulation example is given in Section IV to demonstrate the correctness and effectiveness of our proposed PDSM filtering scheme. Finally, we present the conclusion of this work in Section V.

Notations: The notation used here is fairly standard except where otherwise stated. $\mathbb{R}^{n}$ and $\mathbb{R}^{n \times m}$ denote, respectively, the $n$ dimensional Euclidean space and set of all $n \times m$ real matrices. $\mathbb{N}^{+}$is the set of positive integers. The notation $X \geq Y(X>Y)$, where $X$ and $Y$ are real symmetric matrices, means that $X-Y$ is positive semi-definite (positive definite). $M^{T}$ represents the transpose of matrix $M$. If $A$ is a matrix, $\lambda_{\max }\{A\}\left(\lambda_{\min }\{A\}\right)$ stands for the maximum (minimum) eigenvalue of $A$, and $\operatorname{tr}\{A\}$ denotes the trace of A. 0 represents zero matrix of compatible dimensions. $\mathbf{1}_{N}$ represents an $N$ dimensional row vector with all ones. The $n$-dimensional identity matrix is denoted as $I_{n}$, or simply $I$ if no confusion is caused. The shorthand $\operatorname{diag}\{\cdots\}$ stands for a block-diagonal matrix and the notation $\operatorname{diag}_{n}\{\bullet\}$ is employed to stand for $\operatorname{diag}\{\underbrace{\bullet, \cdots, \bullet}\}$. Given a vector $x,\|x\|$ describes the Euclidean norm of $x$. Matrices, if they are not explicitly specified, are assumed to have compatible dimensions. $\otimes$ is the Kronecker product of matrices. The Kronecker delta function $\delta(a)$ is a binary function that equals 1 if $a=0$ and equals 0 otherwise.

\section{PROBLEM FORMULATION AND PRELIMINARIES}

\section{A. Impulsive measurement outliers}

In this work, we consider the case where the measurements of the sensors could be corrupted by certain outliers.

Obviously, an outlier occurs at one of the sampling instants and therefore the number of occurred outliers should be accountable. In this sense, let $t(i)\left(i \in \mathbb{N}^{+}\right)$denote the occurrence moment of the $i$-th measurement outlier. Based on the sequence $\{t(i)\}_{i \geq 0}$ of the occurrence moments, the measurement outlier $o_{k}$ (i.e., the outlier occurring at the sampling instant $k$ ) can be modeled by the following form of impulsive signals:

$$
o_{k}=\sum_{i=0}^{\infty} \delta(k-t(i)) \hat{o}_{i}
$$

where $\hat{o}_{i}$ represents the amplitude (a vector to be defined later that corresponds to the system measurement) of the $i$-th measurement outlier. For presentation convenience, the measurement outlier $o_{k}$ described in (1) is referred to as an impulsive measurement outlier (IMO). Moreover, by defining the interval length $T_{i}$ as

$$
T_{i}=t(i)-t(i-1)
$$


for $i \in \mathbb{N}^{+}$with initial value $T_{0}=t(0)$, the occurrence moment $t(i)$ can be rewritten as $t(i)=\sum_{j=0}^{i} T_{j}$, which implies that

$$
o_{k}=\sum_{i=0}^{\infty} \delta\left(k-\sum_{j=0}^{i} T_{j}\right) \hat{o}_{i} .
$$

Next, we introduce the following assumption on the proposed IMOs.

Assumption 1: For $\forall i \in \mathbb{N}^{+}$, the interval length $T_{i}$ satisfies $T_{i} \geq \underline{T}$ where $\underline{T}$ is a known positive constant, and the amplitude $\hat{o}_{i}$ satisfies $\left\|\hat{o}_{i}\right\|>\underline{o}$ where $\underline{o}$ is a known constant. We denote $\underline{T}$ and $\underline{o}$ as the lower bounds of the interval length and amplitude, respectively.

Remark 1: It is worth mentioning that Assumption 1 is fairly reasonable in real-world applications. In many practical systems, sensor measurements might suffer from a special class of perturbations which, different from conventional noises, occur on an occasional basis with relatively large amplitudes as compared to the noises. Such occasionally occurred large-amplitude perturbations are modeled as IMOs in this paper whose interval length and amplitude are greater than certain known constants/thresholds that are identifiable from engineering practice or statistical tests. Such outliers are sometimes referred to as "isolated outliers" [46], [47], which means that the outliers appear independently. Obviously, our proposed model (1) is consistent with the description about the isolated outliers. Similar assumption has been adopted in [37], where the moving-horizon estimation problem subject to measurement outliers has been considered and the interval length of two adjacent outliers is assumed to be great than the window/horizon length of the moving-horizon estimation scheme. Obviously, the lower bound of the interval length (i.e. $\underline{T}$ ) is an important index to characterize the occurrence frequency of the IMO. A typical example of such an index can be found in the failure model of a repairable system, where an important index named the time between failures is adopted to model the failure frequency [48], [49].

\section{B. Problem formulation: plant and filter structure}

Consider a discrete linear time-varying system of the form

$$
\left\{\begin{aligned}
x_{k+1} & =A_{k} x_{k}+B_{k} \omega_{k} \\
y_{k} & =C_{k} x_{k}+\nu_{k}+o_{k}
\end{aligned}\right.
$$

where $x_{k} \in \mathbb{R}^{n}$ and $y_{k} \in \mathbb{R}^{m}$ are, respectively, the system state and the measurement output; $\omega_{k} \in \mathcal{W} \triangleq\left\{\omega: \omega^{T} R^{-1} \omega \leq\right.$ $\left.1 ; \omega \in \mathbb{R}^{r}\right\}$ and $\nu_{k} \in \mathcal{V} \triangleq\left\{\nu: \nu^{T} S^{-1} \nu \leq 1 ; \nu \in \mathbb{R}^{s}\right\}$ denote, respectively, the process and measurement noises where $R$ and $S$ are known positive definite matrices with appropriate dimensions; and the parameters $A_{k}, B_{k}, C_{k}$ are real-valued time-varying matrices of appropriate dimensions. Here, the vector $o_{k} \in \mathbb{R}^{m}$ is the IMO of the form (2).

Before proceeding further, we first introduce the following assumptions which are necessary to design a filter for the plant (3).
Assumption 2: There exist positive constants $\bar{a}, \underline{a}, \bar{b}, \underline{b}, \bar{c}$ and $\underline{c}$ such that the following conditions hold for all the $k \geq 0$ :

$$
\begin{aligned}
& \underline{a}^{2} I \leq A_{k} A_{k}^{T} \leq \bar{a}^{2} I, \quad \underline{b}^{2} I \leq B_{k} B_{k}^{T} \leq \bar{b}^{2} I, \\
& \underline{c}^{2} I \leq C_{k} C_{k}^{T} \leq \bar{c}^{2} I .
\end{aligned}
$$

Remark 2: It is worth noting that Assumption 2 guarantees that the matrix $A_{k}$ is invertible and $C_{k}$ is of full row rank. In a great number of real applications, the discrete time-varying system (3) represents the discrete analog of a time-varying continuous-time system subject to certain sampling period. In this case, $A_{k}$ is the state transition matrix which is a timevarying yet invertible matrix. Moreover, $C_{k}$ is a full row rank matrix if the system outputs are measured without redundancy. The condition $B_{k} B_{k}^{T} \geq \underline{b}^{2} I$ means that the matrix $B_{k}$ is of full row rank. Actually, this condition does not lose any generality because, in case it does not hold, we can always apply the following equality to the matrix $B_{k}$

$$
B_{k} \omega_{k}=B_{1, k} B_{2, k} \omega_{k}
$$

where $B_{1, k}$ is a full row rank matrix and $B_{2, k} \omega_{k}$ can be regarded as a new bounded noise satisfying certain ellipsoidal constraint. On the other hand, it is obvious that the system parameters are constrained by certain upper limits in almost all the practical applications. As such, it is quite reasonable to assume that the matrices $A_{k} A_{k}^{T}, B_{k} B_{k}^{T}$ and $C_{k} C_{k}^{T}$ are constrained by certain upper and lower bounds.

Next, let us consider the filter structure for the system (3).We aim to design a recursive set-membership filter for the system (3) where the time-varying filter gain parameter is calculated recursively by certain on-line algorithm. In this paper, the IMO represents the abnormal signal which might result from sensor malfunctions, wrong replacement of measures or large non-Gaussian noises. Note that the filtering performance of the conventional SMF scheme is largely dependent on the bounds of the external inputs (including the outlier signal). Clearly, the addressed IMO is very likely to be unbounded and therefore the conventional SMF scheme would be inapplicable in this case. In order to restrain the filtering performance from being degraded by the outliers, we adopt the following parameter-dependent set-membership (PDSM) filter:

$$
\left\{\begin{aligned}
\hat{x}_{k+1 \mid k} & =A_{k} \hat{x}_{k \mid k} \\
\hat{x}_{k+1 \mid k+1} & =\hat{x}_{k+1 \mid k}+L_{k+1}\left(\theta_{k+1}\right)\left(y_{k+1}-C_{k+1} \hat{x}_{k+1 \mid k}\right) \\
\hat{x}_{0 \mid 0} & =0
\end{aligned}\right.
$$

where $\hat{x}_{k \mid k}$ is the estimate of $x_{k}$ at time instant $k$ with $\hat{x}_{0 \mid 0}=$ 0 , and $\hat{x}_{k+1 \mid k}$ is the one-step prediction at time instant $k$. The PDSM filter parameter $L_{k+1}\left(\theta_{k+1}\right)$ is set to be

$$
L_{k+1}\left(\theta_{k+1}\right)=\left\{\begin{array}{cc}
K_{k+1}, & \text { if } \theta_{k+1}=0 \\
0, & \text { if } \theta_{k+1}=1
\end{array}\right.
$$

where $\theta_{k+1}$ is a binary function to be designed which takes values of 1 and 0 . The matrix $K_{k+1}$ is the time-varying filter parameter to be determined.

Obviously, in the above PDSM filter, the filtering dynamics is largely dependent on the value of $\theta_{k+1}$, which determines 
whether the current innovation should be adopted in calculating the state estimate $\hat{x}_{k+1 \mid k+1}$. More specifically, when $\theta_{k+1}=1$, the value of the state estimate $\hat{x}_{k+1 \mid k+1}$ would be decoupled from the innovation $y_{k+1}-C_{k+1} \hat{x}_{k+1 \mid k}$. In this paper, we aim to design the binary function $\theta_{k+1}$ such that the "harmful innovations" (i.e. the innovations contaminated by IMOs) would be removed. In doing so, the binary function $\theta_{k+1}$ should be designed such that $\theta_{k+1}=1$ if there exists an integer $i \in \mathbb{N}^{+}$satisfying the condition $t(i)=k+1$.

For presentation convenience, we introduce the following definition.

Definition 1: For the time-varying system (3) with filter (4), let the sequence of the constraint matrices (ellipsoid matrices) $P_{k \mid k} \in \mathbb{R}^{n \times n}\left(k \in \mathbb{N}^{+}\right)$be given. The filtering error $e_{k \mid k} \triangleq$ $x_{k}-\hat{x}_{k \mid k}$ is said to satisfy the $P_{k \mid k}$-dependent constraint if the following set of inequalities

$$
e_{k \mid k}^{T} P_{k \mid k}^{-1} e_{k \mid k} \leq 1
$$

holds for $k \in \mathbb{N}^{+}$.

Now, we are ready to state the main objectives of this paper as in the following threefold.

1) Design the binary function $\theta_{k}$ such that the condition $\theta_{k}=1$ holds if and only if $o_{k} \neq 0$ (i.e. there is an outlier occurring at time instant $k$ ). In other words, the designed PDSM filter (4) is capable of removing the innovations contaminated by IMOs.

2) Find a sequence of constraint matrices $\left\{P_{k \mid k}\right\}_{k \geq 0}$ such that the filtering error $e_{k \mid k}$ satisfies the $P_{k \mid k}$-dependent constraint, and then minimize the trace of the matrix $P_{k \mid k}$ by appropriately choosing the filter parameter $K_{k}$ when $\theta_{k}=0$.

3) Analyze the ultimate boundedness of the matrix $\left\{P_{k \mid k}\right\}_{k \geq 0}$.

\section{MAIN RESULTS}

Before proceeding further, let us introduce the following definition and assumption in order to deliver the main results.

Definition 2: [50] Consider the time varying matrices $A_{k}$, $C_{k}$ and let the Observability Gramian be given by

$$
M_{k+N, k}=\sum_{i=k}^{k+N} \Phi_{i, k}^{T} C_{i}^{T} C_{i} \Phi_{i, k}
$$

for the integer $N>0$ with $\Phi_{k, k}=I$ and $\Phi_{i, k}=$ $A_{i-1} A_{i-2} \cdots A_{k}$ for $i>k$. The matrices $A_{k}, C_{k}$ are said to satisfy the uniform observability condition if there are two positive numbers $\underline{r}$ and $\bar{r}$ such that the following inequality holds for the integer $N>0$ :

$$
\underline{r} I \leq M_{k+N, k} \leq \bar{r} I .
$$

Assumption 3: The time-varying matrices $A_{k}$ and $C_{k}$ satisfy the uniform observability condition for all $k>0$ with the integer $N$ and two positive numbers $\underline{r}, \bar{r}$. Furthermore, the minimum interval length $\underline{T}$ of the IMOs satisfies $\underline{T}>N+1$.

\section{A. Design of the function $\theta_{k}$}

In this subsection, we intend to develop a detection method capable of identifying whether the received measurement contains an outlier. First, we would like to establish the inputoutput model of the plant (3) which is to be used when proposing the outlier detection approach. It is easy to see that

$$
\begin{aligned}
\bar{y}_{k-N}^{k} & \triangleq\left[\begin{array}{c}
y_{k-N}-o_{k-N} \\
y_{k-N+1}-o_{k-N+1} \\
\vdots \\
y_{k}-o_{k}
\end{array}\right] \\
& =F_{k} x_{k-N}+G_{k} \omega_{k-N}^{k-1}+\nu_{k-N}^{k}
\end{aligned}
$$

where

$$
\begin{aligned}
& \omega_{k-N}^{k-1} \triangleq\left[\begin{array}{c}
\omega_{k-N} \\
\omega_{k-N+1} \\
\vdots \\
\omega_{k-1}
\end{array}\right], \quad F_{k} \triangleq\left[\begin{array}{c}
C_{k-N} \Phi_{k-N, k-N} \\
C_{k-N+1} \Phi_{k-N+1, k-N} \\
\vdots \\
C_{k} \Phi_{k, k-N}
\end{array}\right], \\
& \nu_{k-N}^{k} \triangleq\left[\begin{array}{cccc}
\nu_{k-N}^{T} & \nu_{k-N+1}^{T} & \cdots & \nu_{k}^{T}
\end{array}\right]^{T}, \\
& G_{k} \triangleq\left[\begin{array}{cccc}
0 & 0 & \cdots & 0 \\
\cdots G_{k}^{\mathrm{T}, \Gamma}-\cdots & 0 & \cdots & 0 \\
G_{k}^{2,1} & G_{k}^{2,2} & \cdots & 0 \\
\vdots & \vdots & \ddots & \vdots \\
G_{k}^{N, 1} & G_{k}^{N, 2} & \cdots & G_{k}^{N, N}
\end{array}\right], \\
& G_{k}^{i, j} \triangleq C_{k-N+i} \Phi_{k-N+i, k-N+j} B_{k-N+j-1} .
\end{aligned}
$$

According to Assumption 3, it is clear that the matrices $A_{k}$ and $C_{k}$ satisfy the condition $\sum_{i=k}^{k+N} \Phi_{i, k}^{T} C_{i}^{T} C_{i} \Phi_{i, k} \geq \underline{r} I$. Then, we have

$$
\begin{aligned}
x_{k-N} & =\left(F_{k}^{T} F_{k}\right)^{-1} F_{k}^{T}\left(\bar{y}_{k-N}^{k}-G_{k} \omega_{k-N}^{k-1}-\nu_{k-N}^{k}\right) \\
& =A_{k-N-1} x_{k-N-1}+B_{k-N-1} \omega_{k-N-1}
\end{aligned}
$$

and therefore

$$
\begin{aligned}
& \bar{F}_{k} \bar{y}_{k-N}^{k}-A_{k-N-1} \bar{F}_{k-1} \bar{y}_{k-N-1}^{k-1} \\
= & \bar{G}_{k} \omega_{k-N}^{k-1}+\bar{F}_{k} \nu_{k-N}^{k}-A_{k-N-1} \bar{G}_{k-1} \omega_{k-N-1}^{k-2} \\
& -A_{k-N-1} \bar{F}_{k-1} \nu_{k-N-1}^{k-1}+B_{k-N-1} \omega_{k-N-1}
\end{aligned}
$$

where

$$
\bar{F}_{k} \triangleq\left(F_{k}^{T} F_{k}\right)^{-1} F_{k}^{T}, \quad \bar{G}_{k} \triangleq\left(F_{k}^{T} F_{k}\right)^{-1} F_{k}^{T} G_{k} .
$$

By defining

$$
\begin{aligned}
& \vec{G}_{k} \triangleq \bar{G}_{k} \mathcal{I}_{1}-A_{k-N-1} \bar{G}_{k-1} \mathcal{I}_{2}, \quad \mathcal{I}_{1} \triangleq\left[\begin{array}{ll}
0 & I_{N} \otimes I_{r}
\end{array}\right], \\
& \vec{F}_{k} \triangleq \bar{F}_{k} \mathcal{I}_{3}-A_{k-N-1} \bar{F}_{k-1} \mathcal{I}_{4}, \quad \mathcal{I}_{2} \triangleq\left[\begin{array}{lll}
I_{N} \otimes I_{r} & 0
\end{array}\right], \\
& \mathcal{I}_{3} \triangleq\left[\begin{array}{lll}
0 & I_{N+1} \otimes I_{m}
\end{array}\right], \quad \mathcal{I}_{4} \triangleq\left[\begin{array}{ll}
I_{N+1} \otimes I_{m} & 0
\end{array}\right],
\end{aligned}
$$

we derive the following equality:

$$
\begin{aligned}
& \bar{F}_{k} \bar{y}_{k-N}^{k}-A_{k-N-1} \bar{F}_{k-1} \bar{y}_{k-N-1}^{k-1} \\
= & \vec{G}_{k} \omega_{k-N-1}^{k-1}+\vec{F}_{k} \nu_{k-N-1}^{k}
\end{aligned}
$$

Note that

$$
\bar{y}_{k-N}^{k}=y_{k-N}^{k}-o_{k-N}^{k}
$$


where $y_{k-N}^{k} \triangleq\left[\begin{array}{llll}y_{k-N}^{T} & y_{k-N+1}^{T} & \cdots & y_{k}^{T}\end{array}\right]^{T}$ and $o_{k-N}^{k} \triangleq$ $\left[\begin{array}{llll}o_{k-N}^{T} & o_{k-N+1}^{T} & \cdots & o_{k}^{T}\end{array}\right]^{T}$. As such, we have the following input-output model for the plant (3)

$$
\vec{F}_{k} y_{k-N-1}^{k}=\vec{G}_{k} \omega_{k-N-1}^{k-1}+\vec{F}_{k} \nu_{k-N-1}^{k}+\vec{F}_{k} \bar{o}_{k-N-1}^{k} \text {. }
$$

Next, let us consider the design problem of the binary function $\theta_{k}$. Based on the input-output model (9), we have the following proposition.

Proposition 1: Under Assumptions 1-3, define the sequence of $\{\hat{t}(i)\}_{i \geq 0}$ as follows:

$\hat{t}(i)= \begin{cases}\min \{k \mid k \geq \underline{T}, f(k)>\bar{f}\}, & \text { if } i=0 \\ \min \{k \mid k \geq \hat{t}(i-1)+\underline{T}, f(k)>\bar{f}\}, & \text { otherwise }\end{cases}$

where

$$
\begin{aligned}
& f(k) \triangleq\left\|\vec{F}_{k} y_{k-N-1}^{k}\right\|, \quad \bar{f} \triangleq \underline{r} \sqrt{\bar{r}}(1+\bar{a})(\bar{g}+\bar{h}), \\
& \bar{g} \triangleq\left\{\begin{array}{ll}
\frac{\bar{c} \bar{b}\left\|U_{1}\right\| \sqrt{\mid N-(N+1) \bar{a}^{2}+\bar{a}^{2(N+1) \mid}}}{\left|1-\bar{a}^{2}\right|}, & \bar{a} \neq 1 \\
\frac{\bar{c} \bar{b}\left\|U_{1}\right\| \sqrt{N(N+1)}}{2}, & \bar{a}=1
\end{array},\right. \\
& \bar{h} \triangleq \sqrt{N+1\left\|U_{2}\right\|,} \quad \vec{f} \triangleq \bar{r}^{-1} \underline{a}^{N} \underline{c},
\end{aligned}
$$

and $U_{i}(i=1,2)$ are the factorizations of $R=U_{1} U_{1}^{T}$ and $S=U_{2} U_{2}^{T}$, respectively. Then, the condition $\hat{t}(i)=t(i)$ holds for all $i \geq 0$ if $\vec{f} \underline{\underline{O}}>2 \vec{f}$.

Proof: The proof of this proposition is performed by mathematical induction as follows.

Initial step. Consider the function $f(k)$. It can be observed that, for all $N+1<\underline{T} \leq k \leq t(0)-1$, we have

$$
f(k)=\left\|\vec{F}_{k} y_{k-N-1}^{k}\right\| \leq\left\|\vec{G}_{k} \omega_{k-N-1}^{k-1}\right\|+\left\|\vec{F}_{k} \nu_{k-N-1}^{k}\right\|
$$

It follows from the definition of $G_{k}$ that

$$
\begin{aligned}
& \left\|G_{k} \omega_{k-N}^{k-1}\right\|=\left\|\left[\begin{array}{c}
0 \\
\sum_{j=1}^{1} G_{k}^{1, j} \omega_{k-N+j-1} \\
\sum_{j=1}^{2} G_{k}^{2, j} \omega_{k-N+j-1} \\
\vdots \\
\sum_{j=1}^{N} G_{k}^{N, j} \omega_{k-N+j-1}
\end{array}\right]\right\| \\
& =\|\left[\begin{array}{c}
0 \\
\sum_{j=1}^{1} G_{k}^{1, j} \omega_{k-N+j-1} \\
0 \\
0 \\
\vdots \\
0
\end{array}\right]+\left[\begin{array}{c}
0 \\
0 \\
\sum_{j=1}^{2} G_{k}^{2, j} \omega_{k-N+j-1} \\
0 \\
\vdots \\
0
\end{array}\right] \\
& +\cdots+\left[\begin{array}{c}
0 \\
0 \\
0 \\
0 \\
\vdots \\
\sum_{j=1}^{N} G_{k}^{N, j} \omega_{k-N+j-1}
\end{array}\right] \| \\
& \leq \sum_{i=1}^{N}\left\|\sum_{j=1}^{i} G_{k}^{i, j} \omega_{k-N+j-1}\right\| \leq \sum_{i=1}^{N} \sum_{j=1}^{i}\left\|G_{k}^{i, j} \omega_{k-N+j-1}\right\|
\end{aligned}
$$

Moreover, it is known from [51] that there exist two vectors $z_{i, k}(i=1,2)$ satisfying $\omega_{k}=U_{1} z_{1, k}$ and $\nu_{k}=U_{2} z_{2, k}$ with $\left\|z_{i, k}\right\| \leq 1$, where $U_{i}(i=1,2)$ are the factorizations of $R=$ $U_{1} U_{1}^{T}$ and $S=U_{2} U_{2}^{T}$, respectively. Then, it is concluded from (11) that

$$
\left\|G_{k} \omega_{k-N}^{k-1}\right\| \leq \sum_{i=1}^{N} \sum_{j=1}^{i} \bar{c} \bar{a}^{i-j} \bar{b}\left\|U_{1}\right\|=\bar{g}
$$

Then, by using (12), we derive that

$$
\left\|\vec{G}_{k} \omega_{k-N-1}^{k-1}\right\| \leq \underline{r} \sqrt{\bar{r}}(1+\bar{a}) \bar{g}
$$

Similarly, we have $\left\|\nu_{k-N}^{k}\right\|^{2} \leq(N+1)\left\|U_{2}\right\|^{2}$ and

$$
\left\|\vec{F}_{k} \nu_{k-N-1}^{k}\right\| \leq \underline{r} \sqrt{\bar{r}}(1+\bar{a}) \bar{h} .
$$

Hence, we have $f(k) \leq \bar{f}$ for all $\underline{T} \leq k \leq t(0)-1$.

On the other hand, under Assumption 1, it is concluded that

$$
\bar{o}_{t(i)-N-1}^{t(i)}=\left[\begin{array}{c}
0_{(N+1) m \times 1} \\
o_{t(i)}
\end{array}\right],
$$

which implies

$$
\begin{aligned}
\vec{F}_{t(i)} \bar{o}_{t(i)-N-1}^{t(i)} & =\bar{F}_{t(i)}\left[\begin{array}{c}
0_{N m \times 1} \\
o_{t(i)}
\end{array}\right]=\left(F_{k}^{T} F_{k}\right)^{-1} F_{k}^{T}\left[\begin{array}{c}
0_{N m \times 1} \\
o_{t(i)}
\end{array}\right] \\
& =\left(F_{k}^{T} F_{k}\right)^{-1} \Phi_{k, k-N}^{T} C_{k}^{T} o_{t(i)} .
\end{aligned}
$$

Hence, we have

$$
\left\|\vec{F}_{t(i)} \bar{o}_{t(i)-N-1}^{t(i)}\right\| \geq \bar{r}^{-1} \underline{a}^{N} \underline{c o}=\overrightarrow{f_{\underline{O}}}
$$

Substituting (15), (13) and (14) into the definition of $f(k)$, we have

$$
\begin{aligned}
& f(t(i))=\left\|\vec{F}_{t(i)} y_{t(i)-N-1}^{t(i)}\right\| \\
= & \left\|\vec{G}_{t(i)} \omega_{t(i)-N-1}^{t(i)-1}+\vec{F}_{t(i)} \nu_{t(i)-N-1}^{t(i)}+\vec{F}_{t(i)} \bar{o}_{t(i)-N-1}^{t(i)}\right\| \\
\geq & \vec{f} \underline{O}-\left\|\vec{G}_{t(i)} \omega_{t(i)-N-1}^{t(i)-1}\right\|-\left\|\vec{F}_{t(i)} \nu_{t(i)-N-1}^{t(i)}\right\| \\
\geq & \vec{f} \underline{O}-\underline{r} \sqrt{\bar{r}}(1+\bar{a})(\bar{g}+\bar{h})>\bar{f}
\end{aligned}
$$

As such, it follows immediately from (16) that

$$
\hat{t}(0)=\min \{k \mid k \geq \underline{T}, f(k)>\bar{f}\}=t(0),
$$

which indicates that the assertion $\hat{t}(i)=t(i)$ holds for $i=0$.

Inductive step. Now that the assertion is true for $i=0$. Next, given that the assertion is true for $i=j$ (i.e. $\theta_{t(j)}=1$ ), we aim to show that the same assertion is true for $i=j+1$.

Similar to the previous deduction, we have $f(t(j+1))>\vec{f}$. On the other hand, for any $t(j)+N+1<t(j)+\underline{T} \leq k \leq$ $t(j+1)-1$, we have $\bar{o}_{k-N-1}^{k}=0$, which implies that

$$
f(k)=\left\|\vec{F}_{k} y_{k-N-1}^{k}\right\| \leq\left\|\vec{G}_{k} \omega_{k-N-1}^{k-1}\right\|+\left\|\vec{F}_{k} \nu_{k-N-1}^{k}\right\| \leq \bar{f} .
$$

Moreover, we have

$$
o_{t(j+1)-N-1}^{t(j+1)}=\left[\begin{array}{c}
o_{t(j+1)} \\
0 \\
\vdots \\
0
\end{array}\right],
$$


which means that

$$
\begin{aligned}
& f(t(j+1))=\left\|\vec{F}_{t(j+1)} y_{t(j+1)-N-1}^{t(j+1)}\right\| \\
= & \| \vec{G}_{t(j+1)} \omega_{t(j+1)-N-1}^{t(j+1)-1}+\vec{F}_{t(j+1)} \nu_{t(j+1)-N-1}^{t(j+1)} \\
& +\vec{F}_{t(j+1)} \bar{o}_{t(j+1)-N-1}^{t(j+1)} \| \\
\geq & -\left\|\vec{G}_{t(j+1)} \omega_{t(j+1)-N-1}^{t(j+1)-1}+\vec{F}_{t(j+1)} \nu_{t(j+1)-N-1}^{t(j+1)}\right\| \\
& +\left\|\vec{F}_{t(j+1)} \bar{o}_{t(j+1)-N-1}^{t(j+1)}\right\| \\
\geq & -\vec{f}+\vec{f}_{\underline{O}}>\bar{f}
\end{aligned}
$$

As such, it can be immediately known from (17) and (18) that

$$
\begin{aligned}
\hat{t}(j+1) & =\min \{k \mid k \geq \hat{t}(j)+\underline{T}, f(k)>\bar{f}\} \\
& =\min \{k \mid k \geq t(j)+\underline{T}, f(k)>\bar{f}\}=t(j+1) .
\end{aligned}
$$

Hence, by the induction, we conclude that the assertion $\hat{t}(i)=$ $t(i)$ is true for $i=0,1, \cdots$. The proof is now complete.

Remark 3: In Proposition 1, we have established a method to derive the sequence of the occurrence moments of outliers based on the input-output model (9). Considering the condition $\vec{f} \underline{O}>2 \bar{f}$, it is easy to conclude that the method proposed in Proposition 1 is effective for "large outliers". In practical applications, the approach presented in Proposition 1 can be utilized to obtain the sequence of the occurrence moments for large outliers while the "small outliers" can be regarded as a class of norm-bounded noises. Obviously, the corresponding measurements contaminated by small outliers would not dramatically deteriorate the filtering performance even if these measurements are involved in the filtering process, which implies that the constructed PDSM filter (4) is still effective to guarantee the desired filtering performance by selecting the suitable filtering parameter.

By means of Proposition 1, we propose an algorithm (i.e. Algorithm 1) to compute the sequence $\{\hat{t}(i)\}_{i \geq 0}$.

Algorithm 1:

Step 1. Initialization: let $i=0$. According to Proposition 1, compute the value of $\bar{f}$. If the current time instant $k<\underline{T}$, wait for the next time instant. Otherwise, go to the next step.

Step 2. Based on the received measurement outputs $\left\{y_{j}\right\}_{k-N-1 \leq j \leq k}$, compute the value of the function $f(k)$.

Step 3. If $f(k)>\bar{f}$, set $\hat{t}(i)=k$. Let $i=i+1$ and go to the next step.

Step 4. If the current time instant $k<\hat{t}(i-1)+\underline{T}$, wait for the next time instant. Otherwise, go to Step 3 .

By employing Algorithm 1, the sequence of the occurrence moments of the outliers is derived by computing the values of $\{\hat{t}(i)\}_{i \geq 0}$ recursively. Based on the acquired sequence $\{\hat{t}(i)\}_{i \geq 0}$, the binary function $\theta_{k}$ is designed as follows:

$$
\theta_{k}= \begin{cases}1, & \text { if }\{i \geq 0 \mid k=\hat{t}(i)\} \neq \emptyset \\ 0, & \text { otherwise }\end{cases}
$$

which means that the condition $\theta_{k}=1$ holds if and only if $o_{k} \neq 0$.

Remark 4: By now, we have completed the design of the binary function $\theta_{k}$. By setting the values of $\left\{\theta_{k}\right\}_{k \geq 0}$ according to Proposition 1, we are capable of identifying whether the received measurement output contains an impulsive outlier, which implies that the first objective of this paper is achieved. The proposed design method of $\theta_{k}$ is implemented based on Assumption 1 and the condition $\vec{f} \underline{\underline{O}}>2 \bar{f}$, which indicates that shortest occurrence period and the smallest amplitude of the impulsive outliers should be larger than certain thresholds to ensure the identifiability of all IMOs. One of our future research topics would be to extend the design method of $\theta_{k}$ to the intermittent outliers.

\section{B. Design of the filter parameter $K_{k}$}

Now, let us consider the filtering error dynamics. Assuming that the condition $\vec{f} \underline{O}>2 \bar{f}$ holds for all $k \geq 0$, we denote the one-step prediction error as

$$
e_{k+1 \mid k} \triangleq x_{k+1}-\hat{x}_{k+1 \mid k} .
$$

Subtracting (4) from (3), we have

$$
e_{k+1 \mid k}=A_{k} e_{k \mid k}+B_{k} \omega_{k}
$$

Similarly, the filtering error dynamics is given as follows:

$$
e_{k+1 \mid k+1}= \begin{cases}\mathcal{K}_{k+1} e_{k+1 \mid k}-K_{k+1} \nu_{k+1}, & \text { if } \theta_{k+1}=0 \\ e_{k+1 \mid k}, & \text { if } \theta_{k+1}=1\end{cases}
$$

where $\mathcal{K}_{k+1} \triangleq I-K_{k+1} C_{k+1}$.

In light of (20) and (21), the constraint matrices are calculated in the following theorem.

Theorem 1: Consider the one-step prediction error dynamics (20) and filtering error dynamics (21). Suppose that the initial state of the plant satisfies $x_{0}^{T} P_{0 \mid 0}^{-1} x_{0} \leq 1$ where $P_{0 \mid 0}$ is a known positive definite matrix. Let $\varepsilon_{1}$ and $\varepsilon_{2}$ be positive scalars. Calculate two sequences of matrices $\left\{P_{k+1 \mid k}\right\}_{k \geq 0}$ and $\left\{P_{k+1 \mid k+1}\right\}_{k \geq 0}$ according to the following difference equations:

$$
\begin{aligned}
& P_{k+1 \mid k}=\left(1+\varepsilon_{1}\right) A_{k} P_{k \mid k} A_{k}^{T}+\left(1+\varepsilon_{1}^{-1}\right) B_{k} R B_{k}^{T} \\
& P_{k+1 \mid k+1} \\
= & \left\{\begin{array}{cc}
P_{k+1 \mid k}, & \theta_{k+1}=1 \\
\left(1+\varepsilon_{2}\right) \mathcal{K}_{k+1} P_{k+1 \mid k} \mathcal{K}_{k+1}^{T} & \theta_{k+1}=0 \\
+\left(1+\varepsilon_{2}^{-1}\right) K_{k+1} S K_{k+1}^{T},
\end{array}\right.
\end{aligned}
$$

Then, the filtering error $e_{k \mid k}$ satisfies the $P_{k \mid k}$-dependent constraint subject to the constraint matrices $\left\{P_{k \mid k}\right\}_{k \geq 0}$. Moreover, when $\theta_{k+1}=0$, the trace of the constraint matrix $P_{k+1 \mid k+1}$ is minimized by the following filter parameter:

$$
K_{k+1}=\left(1+\varepsilon_{2}\right) P_{k+1 \mid k} C_{k+1}^{T} \Omega_{k+1}^{-1}
$$

where $\Omega_{k+1} \triangleq\left(1+\varepsilon_{2}\right) C_{k+1} P_{k+1 \mid k} C_{k+1}^{T}+\left(1+\varepsilon_{2}^{-1}\right) S$.

Proof: The proof of this theorem is carried out by mathematical induction.

Initial step. For $k=0$, it can be immediately known from the initial condition of the plant that

$$
e_{0}^{T} P_{0 \mid 0}^{-1} e_{0}=x_{0}^{T} P_{0 \mid 0}^{-1} x_{0} \leq 1
$$

Inductive step. Now that the assertion is true for $k=0$. Next, given that the assertion is true for $k=i$ (i.e. the filtering error $e_{i \mid i}$ satisfies the condition $e_{i \mid i}^{T} P_{i \mid i}^{-1} e_{i \mid i} \leq 1$ ), we aim to show that $e_{i+1 \mid i+1}^{T} P_{i+1 \mid i+1}^{-1} e_{i+1 \mid i+1} \leq 1$. 
Since the condition $e_{i \mid i}^{T} P_{i \mid i}^{-1} e_{i \mid i} \leq 1$ holds, we have

$$
\left[\begin{array}{cc}
-1 & e_{i \mid i}^{T} \\
* & -P_{i \mid i}
\end{array}\right]<0
$$

which implies that

$$
e_{i \mid i} e_{i \mid i}^{T} \leq P_{i \mid i}
$$

On the other hand, it follows from (20) and (26) that

$$
\begin{aligned}
& e_{i+1 \mid i} e_{i+1 \mid i}^{T}=\left(A_{i} e_{i \mid i}+B_{i} \omega_{i}\right)\left(A_{i} e_{i \mid i}+B_{i} \omega_{i}\right)^{T} \\
= & A_{i} e_{i \mid i} e_{i \mid i}^{T} A_{i}^{T}+B_{i} \omega_{i} \omega_{i}^{T} B_{i}^{T}+A_{i} e_{i \mid i} \omega_{i}^{T} B_{i}^{T}+B_{i} \omega_{i} e_{i \mid i}^{T} A_{i}^{T} \\
\leq & \left(1+\varepsilon_{1}\right) A_{i} e_{i \mid i} e_{i \mid i}^{T} A_{i}^{T}+\left(1+\varepsilon_{1}^{-1}\right) B_{i} \omega_{i} \omega_{i}^{T} B_{i}^{T} \\
\leq & \left(1+\varepsilon_{1}\right) A_{i} P_{i \mid i} A_{i}^{T}+\left(1+\varepsilon_{1}^{-1}\right) B_{i} R B_{i}^{T} \\
= & P_{i+1 \mid i} .
\end{aligned}
$$

Similarly, we have

$$
\begin{aligned}
e_{i+1 \mid i+1} e_{i+1 \mid i+1}^{T} \leq & \left(1+\varepsilon_{2}\right) \mathcal{K}_{i+1} e_{i+1 \mid i} e_{i+1 \mid i}^{T} \mathcal{K}_{i+1}^{T} \\
& +\left(1+\varepsilon_{2}^{-1}\right) K_{i+1} S K_{i+1}^{T} \\
\leq & P_{i+1 \mid i+1}
\end{aligned}
$$

which implies that

$$
e_{i+1 \mid i+1}^{T} P_{i+1 \mid i+1}^{-1} e_{i+1 \mid i+1} \leq 1
$$

Hence, by the induction, it can be concluded that the filtering error $e_{k \mid k}$ satisfies the $P_{k \mid k}$-dependent constraint subject to the constraint matrices $\left\{P_{k \mid k}\right\}_{k \geq 0}$.

Next, let us show that the filter gain given by (24) is optimal in the sense that it minimizes the trace of the constraint matrix $P_{k+1 \mid k+1}$ when $\theta_{k+1}=0$. According to the equation (23), by applying the "completing the square" technique, we have

$$
\begin{aligned}
& \operatorname{tr}\left\{P_{k+1 \mid k+1}\right\} \\
= & \operatorname{tr}\left\{\left(1+\varepsilon_{2}\right) \mathcal{K}_{k+1} P_{k+1 \mid k} \mathcal{K}_{k+1}^{T}+\left(1+\varepsilon_{2}^{-1}\right) K_{k+1} S K_{k+1}^{T}\right\} \\
= & \operatorname{tr}\left\{K_{k+1} \Omega_{k+1} K_{k+1}^{T}-\left(1+\varepsilon_{2}\right) K_{k+1} C_{k+1} P_{k+1 \mid k}\right. \\
& \left.-\left(1+\varepsilon_{2}\right) P_{k+1 \mid k} C_{k+1}^{T} K_{k+1}^{T}+\left(1+\varepsilon_{2}\right) P_{k+1 \mid k}\right\} \\
= & \operatorname{tr}\left\{( K _ { k + 1 } - ( 1 + \varepsilon _ { 2 } ) P _ { k + 1 | k } C _ { k + 1 } ^ { T } \Omega _ { k + 1 } ^ { - 1 } ) \Omega _ { k + 1 } \left(K_{k+1}\right.\right. \\
& \left.-\left(1+\varepsilon_{2}\right) P_{k+1 \mid k} C_{k+1}^{T} \Omega_{k+1}^{-1}\right)^{T}+\left(1+\varepsilon_{2}\right) P_{k+1 \mid k} \\
& \left.-\left(1+\varepsilon_{2}\right)^{2} P_{k+1 \mid k} C_{k+1}^{T} \Omega_{k+1}^{-1} C_{k+1} P_{k+1 \mid k}\right\}
\end{aligned}
$$

Obviously, the trace of the constraint matrix $P_{k+1 \mid k+1}$ is minimized by choosing the filter parameter $K_{k+1}$ according to (24). In this case, the corresponding value of $P_{k+1 \mid k+1}$ is given as follows:

$$
\begin{aligned}
P_{k+1 \mid k+1}= & -\left(1+\varepsilon_{2}\right)^{2} P_{k+1 \mid k} C_{k+1}^{T} \Omega_{k+1}^{-1} C_{k+1} P_{k+1 \mid k} \\
& +\left(1+\varepsilon_{2}\right) P_{k+1 \mid k}
\end{aligned}
$$

The proof is now complete.

Remark 5: So far, we have completed the design issue of the PDSM filter for time-varying systems with impulsive measurement outliers. The computation of $K_{k+1}$ is carried out by solving two discrete-time difference equations, which are suitable for online implementation. It is worth mentioning that the values of the constraint matrices $\left\{P_{k \mid k}\right\}_{k \geq 0}$ are affected by the values of $\left\{\theta_{k}\right\}_{k \geq 0}$ that satisfy the condition $\theta_{k}=1$ for all $o_{k} \neq 0$. As such, the boundedness of constraint matrices $\left\{P_{k \mid k}\right\}_{k \geq 0}$ depends on the sequence of the occurrence moments of outliers (i.e. $\{t(i)\}_{i>0}$ ), which is determined by the sequence of the interval lengths of the outliers (i.e. $\left.\left\{T_{i}\right\}_{i \geq 0}\right)$. In order to guarantee that the filtering algorithm is non-divergent, we shall deal with the boundedness analysis of the constraint matrices $\left\{P_{k \mid k}\right\}_{k \geq 0}$ based on the assumption on the outlier interval length (i.e. Assumption 1) in the next subsection.

\section{Boundedness analysis of constraint matrices}

In this subsection, we would like to consider the boundedness of the constraint matrices $\left\{P_{k \mid k}\right\}_{k \geq 0}$. First, let us give the lower bound of $\left\{P_{k \mid k}\right\}_{k \geq 0}$.

Theorem 2: Under Assumption 2, there exists a positive constant $\underline{p}$ such that the constraint matrix $P_{k \mid k}$ satisfies

$$
P_{k \mid k} \geq \underline{p} I
$$

for every $k>0$, where

$$
\begin{aligned}
& \phi \triangleq\left(1+\varepsilon_{1}^{-1}\right) \lambda_{\min }\{R\} \underline{b}^{2}, \quad \underline{p} \triangleq \min \{\phi, \underline{\phi}\}, \\
& \underline{\phi} \triangleq\left(\phi^{-1}\left(1+\varepsilon_{2}\right)^{-1}+\left(1+\varepsilon_{2}^{-1}\right)^{-1}\left(\lambda_{\min }\{S\}\right)^{-1} \bar{c}^{2}\right)^{-1} .
\end{aligned}
$$

Proof: Let us consider the following two cases:

Case 1: $\theta_{k+1}=0$.

In this case, it follows from (29) that

$$
\begin{aligned}
& P_{k+1 \mid k+1} \\
= & \left(1+\varepsilon_{2}\right) P_{k+1 \mid k}-\left(1+\varepsilon_{2}\right)^{2} P_{k+1 \mid k} C_{k+1}^{T} \Omega_{k+1}^{-1} C_{k+1} P_{k+1 \mid k} \\
= & {\left[\left(1+\varepsilon_{2}\right)^{-1} P_{k+1 \mid k}^{-1}+\left(1+\varepsilon_{2}^{-1}\right)^{-1} C_{k+1}^{T} S^{-1} C_{k+1}\right]^{-1} }
\end{aligned}
$$

On the other hand, we have

$$
P_{k+1 \mid k} \geq\left(1+\varepsilon_{1}^{-1}\right) B_{k} R B_{k}^{T} \geq \phi I
$$

Then, one infers from (31) and (32) that

$$
\begin{aligned}
& P_{k+1 \mid k+1}^{-1} \\
= & \left(1+\varepsilon_{2}\right)^{-1} P_{k+1 \mid k}^{-1}+\left(1+\varepsilon_{2}^{-1}\right)^{-1} C_{k+1}^{T} S^{-1} C_{k+1} \\
\leq & \phi^{-1}\left(1+\varepsilon_{2}\right)^{-1} I+\left(1+\varepsilon_{2}^{-1}\right)^{-1}\left(\lambda_{\min }\{S\}\right)^{-1} \bar{c}^{2} I \\
= & \underline{\phi}^{-1} I
\end{aligned}
$$

which implies $P_{k+1 \mid k+1} \geq \underline{\phi} I$.

Case 2: $\theta_{k+1}=1$.

In this case, we have $P_{k+1 \mid k+1}=P_{k+1 \mid k} \geq \phi I$.

Summarizing the above discussions, it can be concluded that $P_{k+1 \mid k+1} \geq \underline{p} I$. The proof is now complete.

Next, let us consider the upper bound of $\left\{P_{k \mid k}\right\}_{k \geq 0}$. Before proceeding further, we need to do some preparation in the following two propositions.

Proposition 2: Under Assumptions 2-3, we have

$$
\underline{\gamma} I \leq \bar{M}_{k, k-N} \triangleq \sum_{i=k-N}^{k} \Psi_{i, k}^{T} C_{i}^{T} C_{i} \Psi_{i, k} \leq \bar{\gamma} I
$$

for all $k \geq N$, where $\Psi_{k, k}=I$ and

$$
\Psi_{i, k}=A_{i}^{-1} A_{i+1}^{-1} \cdots A_{k-1}^{-1}, \underline{\gamma}=\underline{r} \bar{a}^{-2 N}, \bar{\gamma}=\bar{r} \underline{a}^{-2 N} .
$$


Proof: Obviously, it follows from the definitions of $M_{k, k-N}$ and $\bar{M}_{k, k-N}$ that

$$
\begin{aligned}
& \bar{M}_{k, k-N} \\
= & A_{k-N}^{-T} A_{k-N+1}^{-T} \cdots A_{k-1}^{-T} M_{k, k-N} A_{k-1}^{-1} A_{k-2}^{-1} \cdots A_{k-N}^{-1},
\end{aligned}
$$

which implies

$$
\underline{r} \bar{a}^{-2 N} I \leq \bar{M}_{k, k-N} \leq \bar{r} \underline{a}^{-2 N} I
$$

and the proof is then complete.

Proposition 3: Under Assumptions 2-3 and the proposed PDSM filtering algorithm, there always exists a real constant $0<\beta<1$ such that

$$
P_{k+1 \mid k}^{-1} \geq \beta A_{k}^{-T} P_{k \mid k}^{-1} A_{k}^{-1}
$$

holds for all $k>0$, where

$$
\hbar \triangleq \underline{p}^{-1} \lambda_{\max }\{R\} \bar{b}^{2} \underline{a}^{-2}, \quad \beta \triangleq\left(1+\varepsilon_{1}+\hbar+\varepsilon_{1}^{-1} \hbar\right)^{-1} .
$$

Proof: According to the difference equation (22), we obtain

$$
\begin{aligned}
& P_{k+1 \mid k}^{-1} \\
= & \left(\left(1+\varepsilon_{1}\right) A_{k} P_{k \mid k} A_{k}^{T}+\left(1+\varepsilon_{1}^{-1}\right) B_{k} R B_{k}^{T}\right)^{-1} \\
= & A_{k}^{-T}\left(\left(1+\varepsilon_{1}\right) P_{k \mid k}+\left(1+\varepsilon_{1}^{-1}\right) A_{k}^{-1} B_{k} R B_{k}^{T} A_{k}^{-T}\right)^{-1} A_{k}^{-1}
\end{aligned}
$$

Noting that

$$
A_{k}^{-1} B_{k} R B_{k}^{T} A_{k}^{-T} \leq \lambda_{\max }\{R\} \bar{b}^{2} \underline{a}^{-2} I,
$$

we have $A_{k}^{-1} B_{k} R B_{k}^{T} A_{k}^{-T} \leq \underline{p}^{-1} \lambda_{\max }\{R\} \bar{b}^{2} \underline{a}^{-2} P_{k \mid k}$. Then, it follows from (36) that

$$
\begin{aligned}
P_{k+1 \mid k}^{-1} & \geq A_{k}^{-T}\left(\left(1+\varepsilon_{1}+\hbar+\varepsilon_{1}^{-1} \hbar\right) P_{k \mid k}\right)^{-1} A_{k}^{-1} \\
& =\beta A_{k}^{-T} P_{k \mid k}^{-1} A_{k}^{-1},
\end{aligned}
$$

which concludes the proof.

Now, we are in a position to establish the uniform upper bound of the constraint matrices $\left\{P_{k \mid k}\right\}_{k \geq 0}$.

Theorem 3: Let Assumptions 2-3 hold. Then, there exists a scalar $\bar{p}>0$ such that the constraint matrices $\left\{P_{k \mid k}\right\}_{k \geq 0}$ satisfy the following condition:

$$
P_{k \mid k} \leq \vec{p} I
$$

where

$$
\begin{aligned}
& \bar{p} \triangleq \frac{\bar{a}^{2} \lambda_{\max }\{S\}}{\beta \bar{\varepsilon} \bar{\beta}^{N} \underline{\gamma}}, \bar{\beta} \triangleq\left(1+\varepsilon_{2}\right)^{-1} \beta, \bar{\varepsilon} \triangleq\left(1+\varepsilon_{2}^{-1}\right)^{-1}, \\
& \hat{p} \triangleq \min \left\{\beta \bar{\varepsilon} \bar{\beta}^{N} \lambda_{\max }^{-1}\{S\} \gamma \bar{a}^{-2}, \bar{\beta}^{N} \bar{p}^{-1} \bar{a}^{-2 N}\right\}, \\
& \check{p} \triangleq \min \left\{\beta \bar{\varepsilon} \bar{\beta}^{N} \lambda_{\max }^{-1}\{S\} \gamma \bar{a}^{-2}, \bar{\beta}^{N} \lambda_{\max }^{-1}\left\{P_{0 \mid 0}\right\} \bar{a}^{-2 N}\right\}, \\
& \vec{p} \triangleq \max \left\{\bar{p}, \hat{p}^{-1}, \check{p}^{-1}, \lambda_{\max }\left\{P_{0 \mid 0}\right\}\right\} .
\end{aligned}
$$

Proof: To analyze the boundedness of the constraint matrices $\left\{P_{k \mid k}\right\}_{k \geq 0}$, we consider the following two cases.

Case 1: $\theta_{k+1}=0$.

In this case, we have

$$
\begin{aligned}
P_{k+1 \mid k+1}^{-1}= & \left(-\left(1+\varepsilon_{2}\right)^{2} P_{k+1 \mid k} C_{k+1}^{T} \Omega_{k+1}^{-1} C_{k+1} P_{k+1 \mid k}\right. \\
& \left.+\left(1+\varepsilon_{2}\right) P_{k+1 \mid k}\right)^{-1}
\end{aligned}
$$

$$
=\left(1+\varepsilon_{2}\right)^{-1} P_{k+1 \mid k}^{-1}+\left(1+\varepsilon_{2}^{-1}\right)^{-1} C_{k+1}^{T} S^{-1} C_{k+1}
$$

Then, it follows from (35) and (38) that

$$
P_{k+1 \mid k+1}^{-1} \geq \bar{\beta} A_{k}^{-T} P_{k \mid k}^{-1} A_{k}^{-1}+\left(1+\varepsilon_{2}^{-1}\right)^{-1} C_{k+1}^{T} S^{-1} C_{k+1}
$$

Case 2: $\theta_{k+1}=1$.

In this case, we have

$$
P_{k+1 \mid k+1}^{-1}=P_{k+1 \mid k}^{-1} \geq \beta A_{k}^{-T} P_{k \mid k}^{-1} A_{k}^{-1}
$$

According to the inequality (39), for any $i \geq 1$, it can be derived that

$$
\begin{aligned}
& P_{\bar{k}+1 \mid \bar{k}+1}^{-1} \\
\geq & \bar{\beta} A_{\bar{k}}^{-T} P_{\bar{k} \mid \bar{k}}^{-1} A_{\bar{k}}^{-1}+\left(1+\varepsilon_{2}^{-1}\right)^{-1} C_{\bar{k}+1}^{T} S^{-1} C_{\bar{k}+1} \geq \cdots \\
\geq & \bar{\varepsilon} \sum_{i=0}^{\mu} \bar{\beta}^{i} \Psi_{\bar{k}+1-i, \bar{k}+1}^{T} C_{\bar{k}+1-i}^{T} S^{-1} C_{\bar{k}+1-i} \Psi_{\bar{k}+1-i, \bar{k}+1} \\
& +\bar{\beta}^{\mu+1} \Psi_{\bar{k}-\mu, \bar{k}+1}^{T} P_{\bar{k}-\mu \mid \bar{k}-\mu}^{-1} \Psi_{\bar{k}-\mu, \bar{k}+1}
\end{aligned}
$$

where $\bar{k} \triangleq t(i)-2$ and $\mu \triangleq \bar{k}-t(i-1)$. Noting that $\mu=$ $t(i)-t(i-1)-2 \geq N$, we have

$$
P_{t(i)-1 \mid t(i)-1}^{-1}>\bar{\varepsilon} \bar{\beta}^{N} \lambda_{\max }^{-1}\{S\} \underline{\gamma} I
$$

Then, it follows from (22), (10) and (42) that

$$
\begin{aligned}
P_{t(i) \mid t(i)}^{-1} & \geq \beta A_{t(i)-1 \mid t(i)-1}^{-T} P_{t(i)-1 \mid t(i)-1}^{-1} A_{t(i)-1 \mid t(i)-1}^{-1} \\
& >\beta \bar{\varepsilon} \bar{\beta}^{N} \lambda_{\max }^{-1}\{S\} \underline{\gamma}^{-2} I
\end{aligned}
$$

Hence, we obtain

$$
P_{t(i) \mid t(i)}<\frac{\bar{a}^{2} \lambda_{\max }\{S\}}{\beta \bar{\varepsilon} \bar{\beta}^{N} \underline{\gamma}} I
$$

for all $i \geq 1$. Similarly, it is easy to see that the inequality (44) also holds for $i=0$, which implies that $P_{t(i) \mid t(i)}<\bar{p} I$ for all $i \geq 0$.

On the other hand, for all $t(i) \leq k<t(i+1)(i=0,1, \cdots)$, we have

$$
\begin{aligned}
& P_{k+1 \mid k+1}^{-1} \\
\geq & \bar{\varepsilon} \sum_{i=0}^{k-t(i)} \bar{\beta}^{i} \Psi_{k+1-i, k+1}^{T} C_{k+1-i}^{T} S^{-1} C_{k+1-i} \Psi_{k+1-i, \bar{k}+1} \\
& +\bar{\beta}^{k-t(i)+1} \Psi_{t(i), k+1}^{T} P_{t(i) \mid t(i)}^{-1} \Psi_{t(i), k+1} \\
\geq & \begin{cases}\beta \bar{\varepsilon} \bar{\beta}^{N} \lambda_{\max }^{-1}\{S\} \gamma \bar{a}^{-2} I & \text {, if } k-t(i) \geq N \\
\bar{\beta}^{N} \Psi_{t(i), k+1}^{T} P_{t(i) \mid t(i)}^{-1} \Psi_{t(i), k+1} & , \text { otherwise }\end{cases}
\end{aligned}
$$

Noting that

$$
\bar{\beta}^{N} \Psi_{t(i), k+1}^{T} P_{t(i) \mid t(i)}^{-1} \Psi_{t(i), k+1}>\bar{\beta}^{N} \bar{p}^{-1} \bar{a}^{-2 N},
$$

we have

$$
P_{k+1 \mid k+1} \leq \hat{p}^{-1} I
$$

for all $t(i) \leq k<t(i+1)(i=0,1, \cdots)$. Moreover, for $0<k<t(0)$, it is easy to see that

$$
P_{k \mid k}^{-1} \geq \bar{\beta} A_{k-1}^{-T} P_{k-1 \mid k-1}^{-1} A_{k-1}^{-1} \geq \cdots
$$




$$
\geq \begin{cases}\beta \bar{\varepsilon} \bar{\beta}^{N} \lambda_{\max }^{-1}\{S\} \underline{\gamma} \bar{a}^{-2} I & , \text { if } k \geq N \\ \bar{\beta}^{k} \Psi_{0, k}^{T} P_{0 \mid 0}^{-1} \Psi_{0, k} & , \text { otherwise }\end{cases}
$$

which implies

$$
P_{k \mid k} \leq \check{p}^{-1} I
$$

for all $0<k<t(0)$. Hence, it follows from (45) and (46) that the constraint matrices $\left\{P_{k \mid k}\right\}_{k \geq 0}$ satisfy the following inequality

$$
P_{k \mid k} \leq \vec{p} I,
$$

which is identical to (37). The proof is now complete.

Remark 6: So far, we have studied the SMF problem for linear time-varying systems subject to impulsive measurement outliers. Clearly, the following important factors contribute much to the system complexity: 1) the time-varying system parameters; 2) the noise information (characterized by the matrices $R$ and $S$ ); 3) the shortest occurrence period and the smallest amplitude of the impulsive outliers; and 4) the uniform observability condition of the plant. As expected, all these factors are reflected in the main results concerning the outlier detection scheme, filter parameter design, and boundedness analysis of the filtering error.

Remark 7: In this paper, a systematic investigation is initiated on the new yet challenging problem of set-membership filtering for time-varying systems with measurement outliers. The main novelties of this paper are outlined as follows: 1) the established model of the measurement outlier is new that is in accordance with the engineering practice; 2) a new algorithm is proposed to distinguish the measurement outputs contaminated by outliers from those normal measurements; 3) a new set-membership filter is designed to resist the measurement outliers with guaranteed filtering performance; 4) a rigorous analysis is carried out on the ultimate boundedness issue of the time-varying ellipsoidal region containing the true system state; and 5) the developed filter design algorithm is recursive that is suitable for online applications.

\section{AN ILLUSTRATIVE EXAMPLE}

A numerical example is provided in this section to illustrate the effectiveness of the proposed filter design scheme.

To make our simulation nontrivial, we consider an unstable linear time-varying system of the form (3) with the following time-varying parameters:

$$
A_{k}=\left[\begin{array}{cc}
0 & 1.02+0.1 \sin ^{2}(0.3 k) \\
1.01 & 0
\end{array}\right], B_{k}=I, C_{k}=\left[\begin{array}{ll}
1 & 0
\end{array}\right] .
$$

Then, it is easy to see that

$$
A_{k} A_{k}^{T}=\operatorname{diag}\left\{\left(1.02+0.1 \sin ^{2}(0.3 k)\right)^{2}, 1.01^{2}\right\},
$$

which implies that

$$
1.01^{2} I \leq A_{k} A_{k}^{T} \leq 1.12^{2} I .
$$

Moreover, by setting $N=1$, we have

$$
I \leq M_{k+1, k}=\sum_{i=k}^{k+1} \Phi_{i, k}^{T} C_{i}^{T} C_{i} \Phi_{i, k} \leq 1.12^{2} I .
$$

Meanwhile, it follows from the values of $B_{k}$ and $C_{k}$ that

$$
B_{k} B_{k}^{T}=I, \quad C_{k} C_{k}^{T}=1 .
$$

The process noise is selected as follows:

$$
\omega_{k}=\frac{1}{\sqrt{2}}\left[\begin{array}{l}
\omega_{1, k} \\
\omega_{2, k}
\end{array}\right]
$$

where $\omega_{i, k}(i=1,2)$ is a random variable satisfying $\omega_{i, k}^{2} \leq$ 1. The measurement noise is a random bounded disturbance satisfying $\nu_{k}^{2} \leq 4$. Hence, we obtain $R=I$ and $S=4$. In this illustrative example, we assume that $\underline{T}=4$ and $t(0)=4$. Then, by applying Proposition 1, we have

$$
\bar{f}=\underline{r} \sqrt{\bar{r}}(1+\bar{a})(\bar{g}+\bar{h})=9.0902 .
$$

Let the lower bound of the impulsive measurement outlier be $\underline{o}=2 \bar{f} / \vec{f}+0.1$. Based on the design method of the binary function $\theta_{k}$ in Proposition 1, the impulsive measurement outliers and the values of $\left\{\theta_{k}\right\}_{k \geq 0}$ are shown in Fig. 1. It can be observed from Fig. 1 that our developed function $\left\{\theta_{k}\right\}_{k \geq 0}$ is capable of identifying whether the received measurement contains an impulsive outlier.
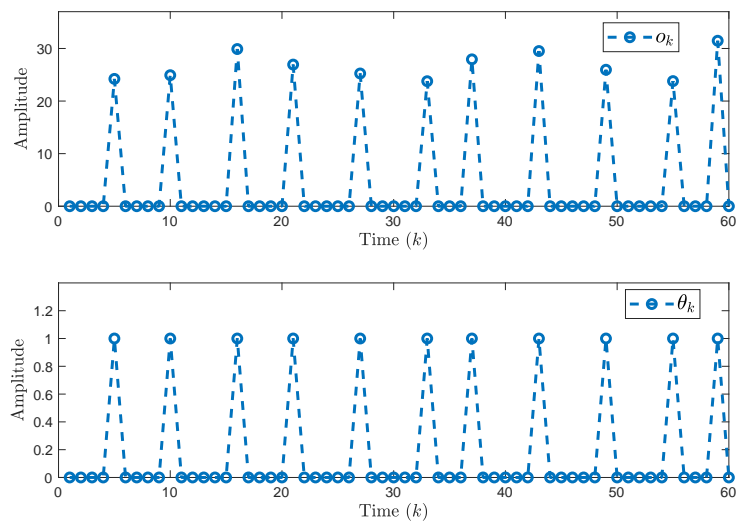

Fig. 1: The measurement outliers and the values of $\left\{\theta_{k}\right\}_{k \geq 0}$

Next, let us try to design the time-varying filter parameter $\left\{K_{k}\right\}_{k>0}$ based on the results in Theorem 1. Set the initial system state as $x_{0}=\left[\begin{array}{ll}7 & 8\end{array}\right]^{T}$. The filtering simulation results are given in Figs. 2-3, which depict the state trajectories and their corresponding estimates. All the simulation results confirm that the filtering performance is well achieved.

In order to show the correctness of the boundedness analysis results about the constraint matrices, Fig. 4 plots the trajectories of the maximum eigenvalues and minimum eigenvalues of $\left\{P_{k \mid k}\right\}_{k \geq 0}$, respectively.

Next, we would like to give a simulation comparison between our developed PDSM filtering scheme and the normal SMF without the outlier detection. The simulation results are given in Fig. 5. It can be easily observed from Fig. 5 that our developed PDSM filtering scheme performs much better than the normal SMF. 


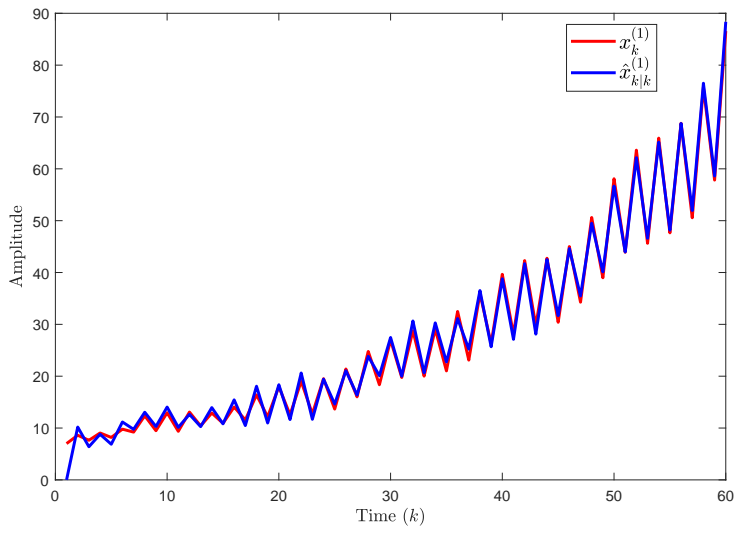

Fig. 2: The state trajectories of $x_{k}^{(1)}$ and $\hat{x}_{k \mid k}^{(1)}$

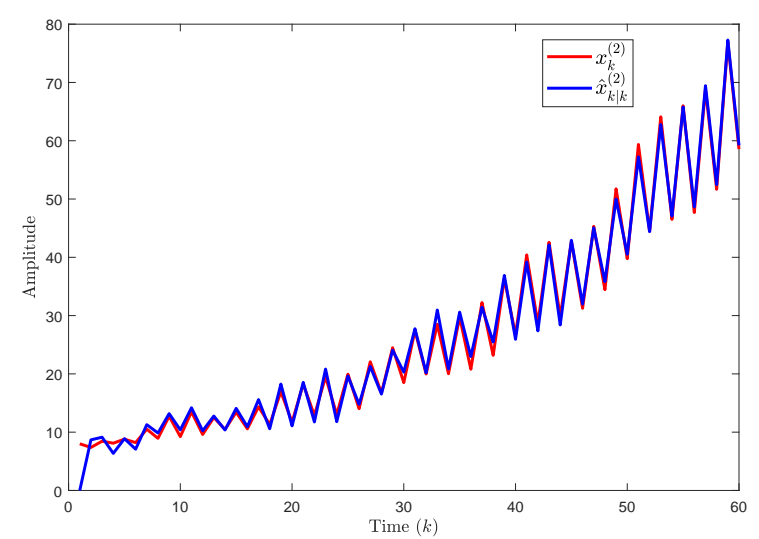

Fig. 3: The state trajectories of $x_{k}^{(2)}$ and $\hat{x}_{k \mid k}^{(2)}$

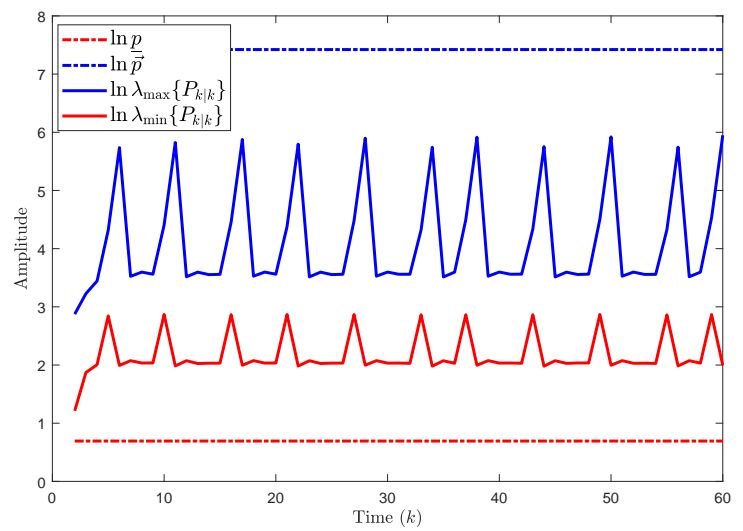

Fig. 4: The trajectories of $\lambda_{\max }\left\{P_{k \mid k}\right\}$ and $\lambda_{\min }\left\{P_{k \mid k}\right\}$

\section{CONCLUSION}

In this paper, the SMF problem has been studied for a class of linear time-varying systems subject to IMOs, which have been modeled by the additive impulsive signals. The interval lengths of outliers (i.e. the times between each two adjacent outliers) are assumed to be larger than certain threshold. Moreover, different from the bounded additive disturbances, the norm of the impulsive measurement outliers is supposed to

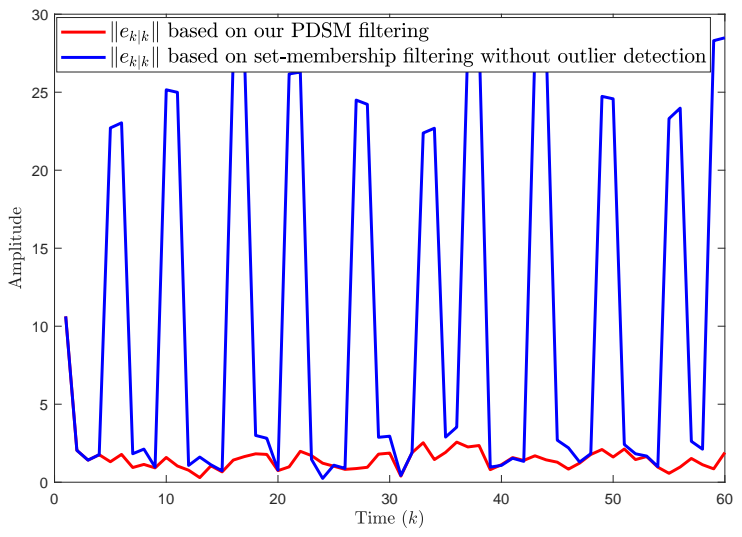

Fig. 5: The trajectories of $\left\|e_{k \mid k}\right\|$ under different filtering approach

be larger than certain known scalar. A special outlier detection approach has been proposed to examine whether the received measurement is corrupted by the impulsive outlier. Based on our provided detection strategy, a PDSM filter has been designed to remove the innovations with outliers. The timevarying filter gain matrix has been derived recursively by solving a class of difference equations. Then, the boundedness analysis issue of the time-varying ellipsoidal region containing the true system state has been studied. Finally, an illustrative example has been provided to demonstrate the effectiveness of our developed PDSM filtering scheme.

Further research topics would include the extension of the main results to 1) the distributed SMF problem for linear systems with IMOs [52], [53], and 2) the PDSM filtering with intermittent measurement outliers [54].

\section{REFERENCES}

[1] M. V. Basin, A. G. Loukianov and M. Hernandez-Gonzalez, Joint state and parameter estimation for uncertain stochastic nonlinear polynomial systems, International Journal of Systems Science, vol. 44, no. 7, pp. 1200-1208, 2013.

[2] R. Caballero-Águila, A. Hermoso-Carazo, and J. Linares-Pérez, Distributed fusion filters from uncertain measured outputs in sensor networks with random packet losses, Information Fusion, vol. 34, pp. 7079, 2017.

[3] H. R. Karimi, and H. Gao, New delay-dependent exponential $H_{\infty}$ synchronization for uncertain neural networks with mixed time delays, IEEE Transactions on Systems, Man, and Cybernetics, Part B-Cybernetics, vol. 40, no. 1, pp. 173-185, Feb. 2010.

[4] W. Li, Y. Jia, and J. Du, Recursive state estimation for complex networks with random coupling strength, Neurocomputing, vol. 219, pp. 1-8, 2017.

[5] D. Liu, Y. Liu and F. Alsaadi, Recursive state estimation based-on the outputs of partial nodes for discrete-time stochastic complex networks with switched topology, Journal of the Franklin Institute, vol. 355, no. 11, pp. 4686-4707, Jul. 2018.

[6] Q. Liu and Z. Wang, Moving-horizon estimation for linear dynamic networks with binary encoding schemes, IEEE Transactions on Automatic Control, in press, DOI: 10.1109/TAC.2020.2996579.

[7] B. Shen, Z. Wang, D. Wang, J. Luo, H. Pu and Y. Peng, Finite-horizon filtering for a class of nonlinear time-delayed systems with an energy harvesting sensor, Automatica, vol. 100, no. 2, pp. 144-152, Feb. 2019.

[8] S. Sun, T. Tan, and H. Lin, Optimal linear estimators for systems with finite-step correlated noises and packet dropout compensations, IEEE Transactions on Signal Processing, vol. 64, no. 21, pp. 5672-5681, 2016.

[9] F. Wang and J. Liang, Constrained $H_{\infty}$ estimation for time-varying networks with hybrid incomplete information, International Journal of Robust and Nonlinear Control, vol. 28, no. 2, pp. 699-715, 2018. 
[10] Y. Xu, R. Lu, P. Shi, H. Li, and S. Xie, Finite-time distributed state estimation over sensor networks with round-robin protocol and fading channels, IEEE Transactions on Cybernetics, vol. 48, no. 1, pp. 336-345, Jan. 2018.

[11] D. Zhang, Z. Xu, H. R. Karimi, and Q. Wang, Distributed filtering for switched linear systems with sensor networks in presence of packet dropouts and quantization, IEEE Transactions on Circuits and Systems I-Regular Papers, vol. 64, no. 10, pp. 2783-2796, 2017.

[12] D. Zhao, S. X. Ding, H. R. Karimi, Y. Li, and Y. Wang, On robust Kalman filter for two-dimensional uncertain linear discrete time-varying systems: A least squares method, Automatica, vol. 99, pp. 203-212, 2019.

[13] L. Zou, Z. Wang, J. Hu and D. Zhou, Moving horizon estimation with unknown inputs under dynamic quantization effects, IEEE Transactions on Automatic Control, in press, DOI: 10.1109/TAC.2020.2968975.

[14] R. Caballero-Águila, I. García-Garrido, and J. Linares-Pérez, Information fusion algorithms for state estimation in multi-sensor systems with correlated missing measurements, Applied Mathematics and Computation, vol. 226, pp. 548-563, 2014.

[15] H. Fang, N. Tian, Y. Wang, M. Zhou and M. A. Haile, Nonlinear bayesian estimation: from Kalman filtering to a broader horizon, IEEE/CAA Journal of Automatica Sinica, vol. 5, no. 2, pp. 401-417, Mar. 2018.

[16] Q. Li, Z. Wang, N. Li and W. Sheng, A dynamic event-triggered approach to recursive filtering for complex networks with switching topologies subject to random sensor failures, IEEE Transactions on Neural Networks and Learning Systems, in press, DOI: 10.1109/TNNLS.2019.2951948.

[17] L. Zou, Z. Wang, Q.-L. Han and D. Zhou, Recursive filtering for timevarying systems with random access protocol, IEEE Transactions on Automatic Control, vol. 64, no. 2, pp. 720-727, Feb. 2019.

[18] N. M. Alyazidi and M. S. Mahmoud, Distributed $H_{2} / H_{\infty}$ filter design for discrete-time switched systems, IEEE/CAA Journal of Automatica Sinica, vol. 7, no. 1, pp. 158-168, Jan. 2020.

[19] Y. Shen, Z. Wang, B. Shen and F. E. Alsaadi, $H_{\infty}$ state estimation for multi-rate artificial neural networks with integral measurements: A switched system approach, Information Sciences, vol. 539, pp. 434-446, Oct. 2020.

[20] Y. Liu, B. Shen and Q. Li, State estimation for neural networks with Markov-based nonuniform sampling: the partly unknown transition probability case, Neurocomputing, vol. 357, pp. 261-270, Sep. 2019.

[21] X. Wan, Z. Wang, M. Wu and X. Liu, State estimation for discrete timedelayed genetic regulatory networks with stochastic noises under the Round-Robin protocols, IEEE Transactions on NanoBioscience, vol. 17, no. 2, pp. 145-154, Apr. 2018.

[22] S. Wang, L. Chen, D. Gu and H. Hu, Cooperative localization of AUVs using moving horizon estimation, IEEE/CAA Journal of Automatica Sinica, vol. 1, no. 1, pp. 68-76, Jan. 2014.

[23] L. Zou, Z. Wang, Q.-L. Han and D. Zhou, Moving horizon estimation for networked time-delay systems under Round-Robin protocol, IEEE Transactions on Automatic Control, vol. 64, no. 12, pp. 5191-5198, Dec. 2019.

[24] L. Zou, Z. Wang, Q.-L. Han and D. Zhou, Moving horizon estimation of networked nonlinear systems with random access protocol, IEEE Transactions on Systems, Man, and Cyberneics-Systems, in press, DOI: 10.1109/TSMC.2019.2918002.

[25] S. Liu, G. Wei, Y. Song and D. Ding, Set-membership state estimation subject to uniform quantization effects and communication constraints, Journal of the Franklin Institute - Engineering and Applied Methematics, vol. 354, no. 15, pp. 7012-7027, Oct. 2017.

[26] F. Yang, N. Xia and Q.-L. Han, Event-based networked islanding detection for distributed solar PV generation systems, IEEE Transactions on Industrial Informatics, vol. 13, no. 1, pp. 322-329, Feb. 2017.

[27] W. Chen, D. Ding, H. Dong and G. Wei, Distributed resilient filtering for power systems subject to denial-of-service attacks, IEEE Transactions on Systems, Man, and Cyberneics-Systems, vol. 49, no. 8, pp. 1688-1697, Aug. 2019.

[28] J. Mao, D. Ding, G. Wei and H. Liu, Networked recursive filtering for time-delayed nonlinear stochastic systems with uniform quantisation under Round-Robin protocol, International Journal of Systems Science, vol. 50, no. 4, pp. 871-884, Mar. 2019.

[29] F. Han, Z. Wang, H. Dong and H. Liu, Partial-nodes-based scalable $H_{\infty}$ consensus filtering with censored measurements over sensor networks, IEEE Transactions on Systems, Man, and Cybernetics-Systems, in press, DOI: 10.1109/TSMC.2019.2907649.
[30] H. S. Witsenhausen, Sets of possible states of linear systems given perturbed observations, IEEE Transactions on Automatic Control, vol. 13, no. 5, pp. 556-558, Oct. 1968.

[31] X. Ge, Q.-L. Han and Z. Wang, A dynamic event-triggered transmission scheme for distributed set-membership estimation over wireless sensor networks, IEEE Transactions on Cybernetics, vol. 49, no. 1, pp. 171183, Jan. 2019.

[32] D. Shi, T. Chen and L. Shi, On set-valued Kalman filtering and its application to event-based state estimation, IEEE Transactions on Automatic Control, vol. 60, no. 5, pp. 1275-1290, May 2015.

[33] G. Wei, S. Liu, Y. Song and Y. Liu, Probability-guaranteed setmembership filtering for systems with incomplete measurements, $\mathrm{Au}$ tomatica, vol. 60, pp. 12-16, Oct. 2015.

[34] N. Xia, F. Yang and Q.-L. Han, Distributed event-triggered networked set-membership filtering with partial information transmission, IET Control Theory and Applications, vol. 11, no. 2, pp. 155-163, Jan. 2017.

[35] F. Yang and Y. Li, Set-membership filtering for discrete-time systems with nonlinear equality constraints, IEEE Transactions on Automatic Control, vol. 54, no. 10, pp. 2480-2486, Oct. 2009.

[36] A. D. Akkaya and M. L. Tiku, Robust estimation in multiple linear regression model with non-Gaussian noise, Automatica, vol. 44, no. 2 , pp. 407-417, Feb. 2008.

[37] A. Alessandri and M. Awawdeh, Moving-horizon estimation with guaranteed robustness for discrete-time linear systems and measurements subject to outliers, Automatica, vol. 67, pp. 85-93, May 2016.

[38] A. Alessandri and L. Zaccarian, Stubborn state observers for linear timeinvariant systems, Automatica, vol. 88, pp. 1-9, Feb. 2018.

[39] A. Gabriel, J. I. Nieto and E. M. Nebot, An outlier-robust Kalman filter, In Proceedings of the 2011 IEEE International Conference on Robotics and Automation, Shanghai, China, pp. 1151-1158, May 2011.

[40] M. A. Gandhi and L. Mili, Robust Kalman filter based on a generalized maximum-likelihood-type estimator, IEEE Transactions on Signal Processing, vol. 58, no. 5, pp. 2509-2520, May 2010.

[41] R. G. Gibbs, New Kalman filter and smoother consistency tests, Automatica, vol. 49, no. 10, pp. 3141-3144, Oct. 2013.

[42] C. Xu, S. Zhao, B. Huang and F. Liu, Distributed Student's t filtering algorithm for heavy-tailed noises, International Journal of Adaptive Control and Signal Processing, vol. 32, no. 6, pp. 875-890, Jun. 2018.

[43] L. Chang and K. Li, Unified form for the robust Gaussian information filtering based on M-estimate, IEEE Signal Processing Letters, vol. 24, no. 4, pp. 412-416, Feb. 2017.

[44] S. C. Chan, Z. G. Zhang and K. W. Tse, A new robust Kalman filter algorithm under outliers and system uncertainties, In Proceedings of the 2005 IEEE International Symposium on Circuits and Systems, Kobe, Japan, pp. 4317-4320, May 2005.

[45] Y. Huang, Y. Zhang, N. Li and J. Chambers, Robust Student's t based nonlinear filter and smoother, IEEE Transactions on Aerospace and Electronic Systems, vol. 52, no. 5, pp. 2586-2596, Oct. 2016.

[46] Z. Sun and H. Sun, Stacked denoising autoencoder with density-grid based clustering method for detecting outlier of wind turbine components, IEEE Access, vol. 7, pp. 13078-13091, 2019.

[47] Z. Yao, J. Xie, Y. Tian and Q. Huang, Using hampel identifier to eliminate profile-isolated outliers in laser vision measurement, Journal of Sensors, vol. 2019, art. no. 3823691, Jul. 2019.

[48] S. Cabuk, Simple test of hypotheses on system availability and mean time to repair, IEEE Transactions on Reliability, vol. 35, no. 5, pp. 581583, Dec. 1986.

[49] Y. Dai, Y. Zhou and Y. Jia, Distribution of time between failures of machining center based on type I censored data, Reliability Engineering and System Safety, vol. 79, no. 3, pp. 377-379, Mar. 2003.

[50] K. Reif, S. Günther, E. Yaz and R. Unbehauen, Stochastic stability of the discrete-time extended Kalman filter, IEEE Transactions on Automatic Control, vol. 44, no. 4, pp. 714-728, Apr. 1999.

[51] L. El Ghaoui and G. Calafiore, Robust filtering for discrete-time systems with bounded noise and parametric uncertainty, IEEE Transactions on Automatic Control, vol. 46, no. 7, pp. 1302-1313, Jul. 2001.

[52] S. Liu, Z. Wang, G. Wei and M. Li, Distributed set-membership filtering for multi-rate systems under the Round-Robin scheduling over sensor networks, IEEE Transactions on Cybernetics, vol. 50, no. 5, pp. 19101920, May. 2020.

[53] N. Xia, F. Yang and Q.-L. Han, Distributed networked set-membership filtering with ellipsoidal state estimations, Information Sciences, vol. 432, pp. 52-62, Mar. 2018.

[54] G. Xiao and F. Liu, Distributed fault-tolerant model predictive control for intermittent faults, IET Control Theory and Applications, vol. 13, no. 10, pp. 1554-1563, Jul. 2019. 


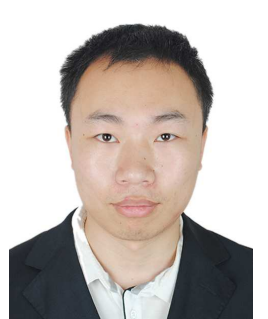

Lei Zou received the B.Sc. degree in automation from Beijing Institute of Petrochemical Technology, Beijing, China, in 2008, the M.Sc. degree in control science and engineering from China University of Petroleum (Beijing Campus), Beijing, China, in 2011 and the Ph.D degree in control science and engineering in 2016 from Harbin Institute of Technology, Harbin, China. From October 2013 to October 2015, he was a visiting Ph.D. student with the Department of Computer Science, Brunel University London, Uxbridge, U.K.

Since 2019, he has been working as a Research Fellow with the Department of Computer Science, Brunel University London, Uxbridge, Uxbridge, U. $\mathrm{K}$. His research interests include control and filtering of networked systems, moving-horizon estimation, and state estimation subject to outliers.

Dr. Zou is currently serving as an Associate Editor for Neurocomputing and International Journal of Systems Science; a Member of IEEE and a Member of Chinese Association of Automation; and a very active reviewer for many international journals.

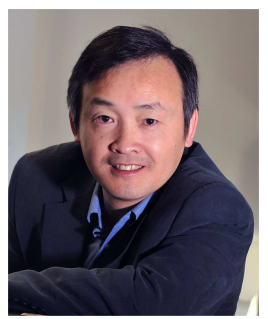

Xiaohui Liu received the B.Eng. degree in computing from Hohai University, Nanjing, China, in 1982 and the Ph.D. degree in computer science from Heriot-Watt University, Edinburg, U.K., in 1988.

$\mathrm{He}$ is currently a Professor of Computing at Brunel University. He leads the Intelligent Data Analysis (IDA) Group, performing interdisciplinary research involving artificial intelligence, dynamic systems, image and signal processing, and statistics, particularly for applications in biology, engineering and medicine. Professor Liu serves on editorial boards of four computing journals, founded the biennial international conference series on IDA in 1995, and has given numerous invited talks in bioinformatics, data mining and statistics conferences.

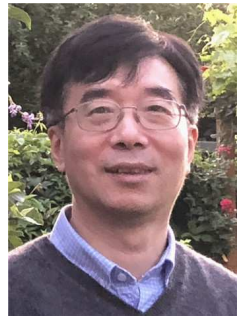

Zidong Wang (SM'03-F'14) was born in Jiangsu, China, in 1966. He received the B.Sc. degree in mathematics in 1986 from Suzhou University, Suzhou, China, and the M.Sc. degree in applied mathematics in 1990 and the Ph.D. degree in electrical engineering in 1994, both from Nanjing University of Science and Technology, Nanjing, China.

$\mathrm{He}$ is currently Professor of Dynamical Systems and Computing in the Department of Computer Science, Brunel University London, U.K. From 1990 to 2002 , he held teaching and research appointments in universities in China, Germany and the UK. Prof. Wang's research interests include dynamical systems, signal processing, bioinformatics, control theory and applications. He has published 250+ papers in IEEE Transactions and $60+$ papers in Automatica. He is a holder of the Alexander von Humboldt Research Fellowship of Germany, the JSPS Research Fellowship of Japan, William Mong Visiting Research Fellowship of Hong Kong.

Prof. Wang serves (or has served) as the Editor-in-Chief for Neurocomputing, the Deputy Editor-in-Chief for International Journal of Systems Science, and an Associate Editor for 12 international journals including IEEE Transactions on Automatic Control, IEEE Transactions on Control Systems Technology, IEEE Transactions on Neural Networks, IEEE Transactions on Signal Processing, and IEEE Transactions on Systems, Man, and CyberneticsPart C. He is a Member of the Academia Europaea, a Fellow of the IEEE, a Fellow of the Royal Statistical Society and a member of program committee for many international conferences.

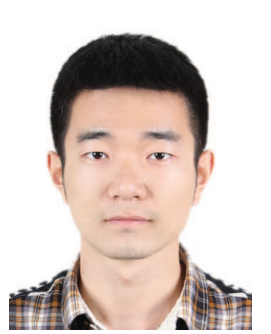

Hang Geng received the Ph.D. degree in Control Science and Engineering from Northwestern Polytechnical University, China, in 2017. From April 2017, he became a Post-Doctoral Fellow with the School of Automation Engineering, University of Electronic Science and Technology of China, China. From March 2018 to March 2019, he was a PostDoctoral Fellow with the Department of Mechanical Engineering, University of Kansas, U.S. He is currently a Research Assistant with the Department of Computer Science, Brunel University London, U.K.

His research interests include information fusion, fault diagnosis and state estimation. 